\title{
Zusammenhang zwischen einer Integrodifferentiàlgleichung und einer Funktionalgleichung
}

\section{FENYö}

Durch Anwendung der Laplace-Transformation wird die Integrodifferentialgleichung

$$
f(t)+\lambda \int_{0}^{-\infty} J_{n}(\dot{2} \sqrt{t x})\left(\frac{t}{x}\right)^{n / 2} f^{(k)}(x) \dot{d} x=h(t)
$$

in eine Funktionalgleichung übergeführt, welche leicht zu lösen ist. Dadurch wird ein Alter-' nàtivsatz, bewiesen und die allgemeine Lösung explizit angegeben.

Прнмененем преобразования Лапласа интегро-дифференциальнос уравнение

$$
f(t)+\lambda \cdot \int_{0}^{\infty} J_{n}(2 \sqrt{t x})\left(\frac{t}{x}\right)^{n / 2} f^{(k)}(x) d x=h(t)
$$

- переводится в фу́нкциональное уравнение, которое легко решается. Этим устанавливается теорема об альтернативе и дается общее решение.

By application of the Laplace-transformation, the integro-differential equation

$$
f(t)+2 \int_{0}^{\infty} J_{n}(2 \sqrt{t x})\left(\frac{t}{x}\right)^{n / 2} f^{(k)}(x) d x=h(t)
$$

can be reduced to a functional equation which can be solved in an easy way. By this idea, an alternative theorem concerning the investigated equation is proved and the general form of solutions is given.

\section{Wir wollen zuerst die Funktionalgleichung}

$$
\left.F(s)+\frac{\lambda}{s^{p}} F\left(\frac{1}{s}\right)=H(s)^{1}\right)
$$

betrachten, wobei $H$ eine gegebene Funktion, $\lambda$ und $p$ gegebene Zahlen und $F$ die unbekannte Funktion ist. Diese Gleichung soll auf einer Teilmenge $D$ der reellen (oder komplexen) Zahlen betrachtet werden, welche mit $s$ auch $1 / s$ enthält. Die Lösung der obigen Funktionalgleichung liefert eine Methode zur Auffindung der Lösungen einer gewissen Integrodifferentialgleichung. Für die Funktionalgleichung (1) gilt der folgende Alternativsatz.

1) Anm. d. Red.: Wir weisen auf die Notiz des Verfassers Solution of an integral equation via functional equation über die entsprechende homogene Funktionalgleichung hin. in: Wiss.

- Beitr. Ingenicurhochschule Wismar, Sonderheft 4 (1985), 18-20. 
Satz A : 1. Ist $\lambda^{2} \neq 1$, so hat Ǵleichung (1)

$$
F(s)=\frac{1}{1-\lambda^{2}}\left[H(s)-\frac{\lambda}{\dot{s}^{p}} H\left(\frac{1}{s}\right)\right]
$$

als einzige Lösung.

2. Ist $\hat{\lambda}^{2}=1$, so hat die inhomogene Gleichung (1) genaiu dann Lösungen, falls $H$ der Gleichung

$$
H(s)=\frac{\lambda}{s^{p}} H\left(\frac{1}{s}\right) \text {, }
$$

genüğt. Ist diese erfüllt, so ist die allgemeine Lösung von (1) von der Gestalt

$$
F(s)=\frac{1}{2}\left[H(s)+\frac{1}{s^{p}} G\left(\frac{1}{s}\right)-\lambda G(s)\right],
$$

wobei $G$ eine beliebige auf $D$ definierte Funktion ist.

3. Ist $\lambda^{2}=1$, so hat die zu (1) gehörige homogene, Gleichung nichttriviale Lösungen, deren allgemeine Gestalt

$$
F(s)=G(s)-\frac{\lambda}{s^{p}} G\left(\frac{1}{s}\right)
$$

ist, wobei $G$ eine beliebige auf $D$ definierte Funktion ist.

1. Aus der Behauptung 1 ergibt sich sofort, daß im Falle $\lambda^{2} \neq 1$ die za (1) gehörige homogene Gleichung nur die triviale Lösung hat. Dies ist ein Spezialfall aus [4: Theorem 2 ]. 2. Satz A hat zum Inhalt, $\operatorname{dia} \lambda \lambda= \pm 1$ die Eigenwerte des Operators $(A f)(s)=1 / s^{p} /(1 / s)$ sind. Dieser ist für alle auf $D$ definierten Funktionen erklärt.

Beweis: 1. Man setze in (1) anstatt $s$ den Ausdruck $1 / s$ ein: $F(1 / s)+\lambda s^{p} F(s)$ $=H(1 / s)$. Eliminiert man hieraus und aus (1) $F(1 / s)$, so ergibt sich

$$
\left(1-\lambda^{2}\right) H(s)=H(s)-\frac{\lambda}{s^{p}} H\left(\frac{1}{s}\right)
$$

woraus als einzige Iösung (2) hervorgeht.

2. Aus der Beziehung (6) sieht man sofort, daß im Falle $\lambda^{2}=1$ für die Existenz der Lösung die Beziehung (3) notwendig ist. Sie ist aber auch hinreichend. Um das für den Fall $\lambda \doteq 1$ zu zeigen (für den Fall $\lambda=-1$ verläuft der Beweis ganz analog), betrachten wir die abgeänderte. Funktionalgleichung

$$
Z_{a}(s)+\frac{1+a}{s^{p}} Z_{a}^{\prime}\left(\frac{1}{s}\right)=H(s)+u G(s) \quad(s \in D),
$$

wobei $a \neq 0,2$, sonst aber eine beliebige Zahl und $G$ eine auf $D$ definierte beliebige Funktion ist. (7) ist eine inhomogene Funktionalgleichung von der Gestalt (1), wobei für den Parameterwert $(1+a)^{2} \neq 1$ ist. Somit hat (7) nach (2) die einzige Iösung

$$
\begin{aligned}
Z_{a}(s)= & -\frac{1}{a(a+2)} H(s)+\frac{1+a}{a(a+2)} \frac{1}{s^{p}} H\left(\frac{1}{s}\right) \\
& -a G(s)+\frac{1+a}{a+2} \frac{1}{s^{p}} G\left(\frac{1}{s}\right) .
\end{aligned}
$$


Wir nehmen jetzt an, da $B H$ der Bedingung (3) mit $\lambda=1$ genügt. Uniter Beriicksichtigung dieser Beziehung ergibt sich

$$
Z_{a}(s)=\frac{1}{a+2}\left[H(s)-G(s)+\frac{1+a}{s^{p}} \dot{G}\left(\frac{1}{s}\right)\right] .
$$

Bei $a \nrightarrow 0$ geht (7) in $_{c}(1)$ und $Z_{a}$ in den Ausdruck (4) über. Dieser Schritt ist legal, da $Z_{a}$ für jedes feste's $s$ eine stetige Funktion im Punkte $a=0$ ist. Man sieht auch leicht ein, da B (4) die allgemeine Lösung von (1) ist. Dazu sei $F$ eine beliebige Iösung von (1). Mit dieser kann der Ausdruck (4) jetzt als eine Funktionalgleichung beziiglich $G$ betrachtet werden. Diese ist'genau vom Typ (1). Für diese ist, wie man sofort sieht, die Existenzbedingung (3) erfüllt, was besagt, da $B$ zu jeder 'Lösung $F$ von (1) eine Funktion $G$ derart bestimmt werden kann, da $B$ die Beziehung (4) gilt."

3. (5) ergibt sich unmittelbar aus (4); wenn wir $H=0$ setzen

2. Wir werden jetzt zur Betrachtung der Integrodifferentialgleichung

$$
f(t)+\lambda \int_{0}^{\infty} J_{n}(2 \sqrt{t x})\left(\frac{t}{x}\right)^{n / 2} f^{(k)}(x) d x=h(t)
$$

übergehen. Hier bedeutet $J_{n}$ die Besselsche Funktion erster Art, $h$ eine gegebeñe in $(0, \infty)$ definierte Funktion, $\lambda$ êinen Parameter, $n$ und $k$ nichtnegative ganze Zahlen. Man bemerke, daß der zweite.Summand auf der linken Seite von (8) die (modifizierte) Hankel-Transformierte von $f^{(k)}$ ist. Der Funktionenraum $B_{n}{ }^{k}$, in welchem wir die Lösung : von (8) suchen, bestehe genau aus denjenigen Funktionen $f$ auf $(0, \infty)$; welche mindestens $k$-mal differenzierbar sind (die $(k-1)$-ten Ableitungen seien absolut stetig), für welche die Grenzwerte $f^{(i)}(+0)(j=0,1, \ldots, k-1)$ existieren und (einfachheitshalber) gleich Null sind, für welche $f^{(k)}$ die Hankel-Transformierte $n$-ter Ordnung für fast alle $t>0$ besitzt und $t^{n / 2} f(t)$ Laplace-transformierbar mit . einer Konvergenzabszisse $\leqq 0$ ist.

Hinreichend, da $\beta$ eine Funktion $f$ in $B_{n}{ }^{k}$ sei, ist die Erfüllung folgender Bedingungen:

a) $j \in C^{k}(0, \infty) \cdot f^{(j)}(+0)=0^{\prime}(j=0,1, \ldots, k-1)$,

b) $\int_{0}^{\infty} \mathrm{e}^{-\delta t t^{n / 2}}|f(t)| d t<\infty$ für alle $\delta>0$,

c) $\int_{0}^{\infty} t^{-n^{2}-1 / 2}\left|f^{(k)}\left(\frac{t^{2}}{2}\right)\right| d t<\infty$.

Die Behauptungen'a) und b) sind evident. Wenn wir im Integral von (8) für $t$ die Variable $u=\sqrt{2 t}$ und für $x$ die Veränderliche $v=\sqrt{2 x}$ einführen, so ergibt sich

$$
\int_{0}^{\infty} J_{n}(2 \sqrt{t x})\left(\frac{t}{x}\right)^{n / 2} f^{(k)}(x) d x=\int_{0}^{\infty} J_{n}(u v)\left(\frac{u}{v}\right)^{n} f^{(k)}\left(\frac{v^{2}}{2}\right) d v .
$$

Dann' aber folgt nach einem wohlbekannten Satze (s. z. B. [2: p. 182]) aus c) die Existen' der Hankel-Transformierten für alle $\ell>0$.

Wir werden auch über die willkürliche Funktion $h$ in (8) voraussetzen, daß diese aus $B_{n}{ }^{k}$ ist.

Wir benötigen einen weiteren ebenfalls bekannten Hilfssatz [3: Satz 52/p. 237]. Nach diesem. gilt: Ist eine Fúnktion $\varphi$ laplace-transformierbar, dann ist ihre 
Hankel-Transformierte Laplace-transformierbar und es gilt ( $\operatorname{Re} s>0$ ).

$$
\mathscr{L}\left\{\int_{0}^{\infty} J_{n}(2 \sqrt{t x})\left(\frac{t}{x}\right)^{n / 2} \varphi(x) d x\right\}(s)=\frac{1}{s^{n+1}} \mathscr{L}\{\varphi\}\left(\frac{1}{s}\right) .
$$

Satz B: 1. Ist $\lambda^{2}+1$, so hat Gleichung (8)

$$
f(t)=\frac{1}{1-\lambda^{2}}\left[h(t)-\lambda \int_{0}^{\infty} J_{n}(2 \sqrt{t x})\left(\frac{t}{x}\right)^{n / 2} h^{(k)}(x) d x\right]
$$

als einzige Lösung im Raume $B_{n}{ }^{k}$.

2. Ist $\lambda^{2}=1$, so hat die inhomogene Gleichung (8) genau dann zum Raum $B_{n}{ }^{k}$ gehörige Lösungen, falls $h$ der Gleichung

$$
h(t)=\lambda \int_{0}^{\infty} J_{n}(2 \cdot \sqrt{t x})\left(\frac{t}{x}\right)^{n / 2} h^{(k)}(x) \cdot d x
$$

genügt. Ist diese erfüllt, so ist die allgemeine Lösung von' (8) von der Gestalt

$$
f(t)=\frac{1}{2}\left[h(t)-\lambda g(t)+\int_{0}^{\infty} J_{n}(2 \sqrt{t x})\left(\frac{t}{x}\right)^{n / 2} g^{(k)}(x) d \dot{x}\right],
$$

wobei g eine beliebige Funktion aus $B_{n}{ }^{*}$ ist.

3. Ist $\lambda^{2}=1$, so hat die zu (8) gehörige homogene Gleichung nichttriviale Lösungen, deren allgemeine Gestalt

$$
f(t)=\int_{0}^{\infty} J_{n}(2 \cdot \sqrt{t x})\left(\frac{t}{x}\right)^{n / 2} \cdot g^{(k)}(x) d x-\lambda g(t)
$$

ist, wobei $g$ eine beliebige Finktion aus $B_{n}{ }^{k}$ ist.

Beweis: Wir. setzen zuerst voraus, daß-(8) eine Lösung-aus $B_{n}{ }^{k}$ besitzt. Unter Berücksichtigung der klassischen Formel

$$
\mathscr{L}\left\{\varphi^{(r)}\right\}(s)=s^{r} \mathscr{L}\{\varphi\}(s)-\varphi(+0) s^{r-1}-\varphi^{\prime}(+0) s^{r-2}-\cdots-\varphi^{(r-1)}(+0)
$$

$(r=0,1, \ldots)$ und der Regel (9) erhalten wir durch gliedweise Bildung der LaplaceTransformierten von (8)

$$
F(s)+\frac{\lambda}{s^{n+k+1}} F\left(\frac{1}{s}\right)=H(s) \quad(\operatorname{Re} s>0),
$$

wobei $\mathscr{L}(f)=F$ und $\mathscr{L}(h)=H$ ist. Dabei wurde schon beriicksichtigt, da $B f$ und $h$ aus $B_{n}{ }^{k}$ sind. (14) ist eine Funktionalgleichung genau vom Typ (1), wobei jetźt $D=(0, \infty)$ ist. Wir können dementsprechend den Satz A auf (14). anwenden. Die Auflösung der Integrodifferentialgleichung (8) wurde somit auf die Auflösung der Funktionalg'leichuing (14) reduziert:,

1. Ist $\lambda^{2} \neq 1$, so ergibt sich laut Satz $A / 1$, da $B$ (14) genau cine Lösung hat, und diese ist nach (2)

$$
F(s)=\frac{1}{1-\lambda^{2}}\left[H(s)-\frac{\lambda}{s^{n+k+1}} H\left(\frac{1}{s}\right)\right] .
$$


Da die rechte Seite diesèr Beziehung eine. inverse Laplace-Transformierte hat, . werden wir, nochmals durch Berücksichtigung von (9), die Rücktransformation durchführen und erhalten

$$
f(t)=\frac{1}{1-\lambda^{2}}\left[h(t)-\lambda \int_{0}^{\infty} J_{n}\left(2 \sqrt{\frac{i x}{x}}\right)\left(\frac{t}{x}\right)^{n / 2} h^{(k)}(x) d x\right] .
$$

'Angenommen, daß (8) eine Lösung aus $B_{n}{ }^{k}$ hat, ergab sich, daß diese nur.von obiger Gestalt sein kann. Es läßt sich sofort zeigen, dä $B$ die unter (16) angegebene Funktion die Gleichung (8) tatsächlich befriedigt. Denn unter Berücksichtigung von (9)

: folgt aus (16) die Bezichung (15), welche wiederum mit (14) gleichbedeutend ist. Aus (14) ergibt sich wiederum nach (9)

$$
f(t)+\lambda \int_{0}^{\infty} J_{n+k}(2 \sqrt{t x})\left(\frac{t}{x}\right)^{(n+k) / 2} f(x) d x=h(t)
$$

Man sicht sofort aus (16), daß $f$ beliebig oft differenzierbar ist, daher gilt nach einer bekannten Formel [1: Formel 11.7]

$$
\int_{0}^{\infty} J_{n+k}(2 \sqrt{t x})\left(\frac{t}{x}\right)^{(n+k) / 2} f(x) d x=\int_{0}^{\infty} J_{n}(2 \sqrt{t x})\left(\frac{i}{x}\right)^{n / 2} f^{(k)}(x) d x,
$$

womit wir (8) erhalten haben.

2. Ist $\lambda^{2}=1$, so hat die inhomogene Gleichung (14) nach Satz $A / 2$ genau dann - Lösungen, falls

$$
H(s) \doteq \frac{\lambda}{s^{n+k+1}} H\left(\frac{1}{s}\right) \quad(s>0)
$$

gilt. Daraus folgt unter Beachtung von' (9) die Bedingung (11). Ist umgekehrt (11) erfüllt, so. folgt daraus (17) und nach Satz A/2, daß (14) Lösungen besitzt. Diese haben die Form.

$$
F(s)=\frac{1}{2}\left[\dot{H}(s)+\frac{1}{s^{n+k+1}} G\left(\frac{1}{s}\right)-\lambda G(s)\right]
$$

wobei jètzt $G$ eine Funktion ist, deren inverse Laplace-Transformierte existieit, die sonst aber beliebig ist. Bezeichnet nun $g$ eine beliebige Funktion aus $B_{n}{ }^{k}$ und setzt $\operatorname{man}^{\prime} \mathscr{L}\{g\}=G$, so ergibt sich (12).

3. Setzt man $h=0$, so ergibt sich die Behauptung (13)

1. Aus der Behauptung 1 ergibt sich sofort, da $\beta$ im Falle $\lambda^{2} \neq 1$ die zu. (8) gehörige homo. gene Integrodifferentialgleichung nur die triviale Lösung hat.

2: Wir können die Integrodifferentialgleichung (8) unter etwas allgemeineren Vornussetzun. gen betrachten, indem wir von der Lösung dio Erfüllung der Anfangsbedingungen $f^{(j)}(+0)$ $=0(j=0,1, \ldots, k-1)$ nicht unbedingt fordern. Man ersetżt dann die Bedingung a) durch

$\left.a^{\prime}\right) f \in C^{k}(0, \infty), f^{(j)}(+0)(j=0,1, \ldots, k-1)$ existieren und sind endlich.

Statt der Funktionalgleichung (14) erhält man die folgende:

$$
F^{\prime}(s)+\frac{\lambda}{s^{n+k+1}} F\left(\frac{1}{s}\right) \doteq H(s)+\sum_{j=0}^{k-1} \frac{f^{(j)}(+0)}{8^{k-j+n}} .
$$


Wenn wir diesmal den Satz A auf (14') anwenden, dann \%eigt ein leichtes Rechnen folgendes: $\therefore$ Ist $\hat{\lambda}^{2} \neq 1$, so muß die Lösung die Gestalt

$$
\begin{aligned}
f(t)= & \frac{1}{1-\lambda^{2}}\left[h(t)-\lambda \int_{0}^{\infty} J_{n}(2 \sqrt{t x})\left(\frac{t}{x}\right)^{n / 2} h^{(k)}(x) d x\right. \\
& \left.+\sum_{j=0}^{k-1} f^{(j)}(-j-0) \frac{t^{n+k-j-1}}{(n+k-j-1) !}-\lambda \sum_{j=1}^{k-1} f^{(j)}(+0) \frac{t^{j}}{j !}\right]
\end{aligned}
$$

haben. Die $k$-te Ableitưng der Polynomterme auf der rechten Seite von $\left(16^{\prime}\right)$ können HankelTransformierte nur dann besitzen, wenn entweder (i) $n=0$ oder (ii) $n>0$ und $f^{(k)}(+0)=0$ $(j=0,1, \ldots, m-1)$ zutrifft, wobei $m=\min (n, k)$ ist. Im Falle (i) ist also die Lösung von (8) nebst Anfangsbedingungen $f^{(j)}(+0)=a_{j}\left(j=0,1, \ldots, k-1 ; a_{j}\right.$ sind beliebige Zahlen):

$$
f(t)=\frac{1}{1-\lambda^{2}}\left[h(t)-\lambda \int_{0}^{\infty} J_{0}(2 t x) h^{(k)}(x) d x+\sum_{j=0}^{k-1} a_{j} \frac{t^{k-j-1}}{(k-j-1) !}-\lambda \sum_{j=0}^{k-1} a_{j} \frac{t i}{j !}\right] .
$$

Im Falle (ii) hat die Lösung die Gestalt $\left(16^{\prime}\right)$, in welcher jedoch $f(-t-0)=f^{\prime}(+0)=\cdots$ $=f^{(m)}(+0)=0$ und $f^{(m+1)}(+0)=a_{m+1}, \ldots, f^{\left(k^{\prime}-1\right)}(+0)=a_{k-1}\left(a_{m+1}, \ldots, a_{k-1}\right.$ beliebige Zahlen $)$ gesetzt werden soll.

Ist $\lambda^{2}=1$ und wenden wir Satz A/2 auf $\left(14^{\prime}\right)$ an, so ergibt sich für die Auflösbarkeit von (14') die Bedingung

$$
H(s)+\sum_{j=0}^{k-1} \frac{f^{(j)}(+0)}{s^{k-j-1}}=\frac{\lambda}{s^{n ! k+1}} H\left(\frac{1}{s}\right)+\lambda \cdot \sum_{j=0}^{k-1} \frac{j^{(j)}(+0)}{s^{n+2 k+j}} .
$$

Wenn wir diese als eine Funktionalgleichung bezüglich $H$ betrachten, dann kann letztere, wiederum nach Satz $\mathrm{A} / 2$, genau dann befriedigt werden, wenn für $s>0$

$$
\text { 2. } \sum_{j=0}^{k-1} \frac{f^{(j)}(+0)}{s^{n+2 k+j}}-\sum_{j=0}^{k-1} \frac{f^{(j)}(+0)}{s^{k-j-1}}=\lambda \sum_{j=0}^{k-1} \frac{f^{(j)}(-0)}{s^{k-j-1}}-\sum_{j=0}^{k-1} \frac{f^{(j)}(+0)}{s^{n-2 k+j}}
$$

gilt. Ist $\lambda=1$, dann geht diese Beziehung in eine Identität über. Das bedeutet, falls $h$ der Bedingung (11) (d. h. $H$ der Bedingung'(17)) genügt, daßB dann die Integrodifferentialgleichung (8) mit beliebigen Anfangswerten befriedigt werden kann. Wenn aber $\lambda=-1$ ist, so ist (mit.der Bezeichnung $f^{(j)}(+0)=a_{j}$ )

$$
\sum_{j=0}^{k-1} \frac{a_{j}}{s^{n+2 k+j}}=\sum_{j=0}^{k-1} \frac{a_{j}}{s^{k-j-1}} \quad(s>0) .
$$

Auf der linken und rechten Seite gibt es keine zwei Exponenten von $1 / s$, welche untereinander gleich wären, deshalb kann obige Beziehung für alle positiven Werte von $s$ nur dann gelten, wenn alle $a_{j}$ gleich Null sind. Das bedeutet, daß es für den Eigenwert $\lambda=1$ keine anderen Lösungen von (8) gibt, nur solche, welche dem Raum $B_{n}{ }^{k}$ angehören. -

3. Wenn wir (8) für $k=0$ betrachten, so bedeutet unser Ergebnis, daß die Eigenwerte der ,Hankel-Transformation 1 und -1 sind (was. schon aus der Tatsache hervorgeht, daß die Hankel-Transformation involutorisch ist). Die $z u$ ihnen gehörigen Eigenfunktionen haben die Gestalt

$$
f(t)=\int_{0}^{\infty} J_{n}(2 \sqrt{t x})\left(\frac{t}{x}\right)^{n / 2} g(x) d x \bar{\mp} g(t) \quad\left(g \in B_{n}^{0}\right)
$$


4. Da die Hankel-Transformation involutorisch ist, folgt sofort aus (8)

$$
\lambda f^{(k)}(t)+\int_{0}^{\infty} J_{n}(2 \sqrt{t x})\left(\frac{t}{x}\right)^{n / 2} f(x) d x=\int_{0}^{\infty} J_{n}(2 \sqrt{t x})\left(\frac{t}{x}\right)^{n / 2} h(x) d x=g(x),
$$

wobei jetzt $g$ eine gegebene Funktion bedeutet, deren Hankel-Transformierte für alle $t>0$ existiert. Diese Integrodifferentialgleichung ist mit (8) gleichwertig. ${ }^{2}$ )

2) Der Verfasser möchte auf diese Weise seine Dankbarkeit an den Herrn Referenten, dem er viele wertvolle Hinweise verdankt, zum Ausdruck bringen.

\section{LITERATUR}

[1] Ditkrs, V. A., and A. P. Pridukov: Integral Tränsforms and Operational Calculus. New York: Pergamon Press 1965.

[2] Magnus, W., und F. Oberiettinger: Formeln und Sätze für die speziellen Funktionen der mathematischen Physik. Berlin-Göttingen-Heidelberg: Springér-Verlag 1948.

[3] Merster, E.: Integraltransformationen mit Anwendungen auf Probleme der Mathematischen Physik. Frankfurt/M. - Bern - New York : Peter Lang Verlag 1983.

[4] Przeworska-Rolewicz, D.: Right -invertible operators and functional-differential equations with involutions. Demonstratio Math. $\tilde{5}(1973), \cdot 16 \tilde{5}-177$.

Manuskripteingang: 21.03. 1985; in revidierter Fassung 19.07. 1985

\section{VERFASSER :}

Prof. em. Dr. I.'S. Fenyö

Technische Universität

Mathematisches Institut der Elektrotechnischen Fakultät H.1111 Budapest, Stoczek u. 2 